\title{
PHOTODIMERIZATION OF ANTHRYL GROUPS ATTACHED TO PMMA SIDE CHAIN IN DILUTE SOLUTION
}

\author{
Fumio Nakamura, Yoshihiko Tezuka, Hirofumi Yajima and Tadahiro Ishii \\ Department of Applied Chemistry, Faculty of Science \\ Science University of Tokyo, Kagurazaka, Shinjuku-ku, Tokyo 162 Japan
}

In order to establish a kinetics of the reaction between reactive groups attached to polymer side chains, we have measured the reaction rate of photodimerization of anthryl groups attached in part to polymethylmethacrylate (PMMA) side chains in dilute solutions. A dynamic light scattering (DLS) measurement and a gel permeation chromatography (GPC) have been used to estimate the volume of an individual polymer coil. At the initial stage of the reaction, the volume does not change so greatly and the reaction obeys a second order kinetics in the same manner as a monomer-like model compound. When the reaction proceeds up to some extent, the volume decreases rapidly and the reaction is restricted to deviate from the second order kinetics

\section{Introduction}

Reactions between reactive groups attached to a polymer chain are different from those of the corresponding small molecules. They are greatly influenced by conformation of the polymer chain and its dynamic motion. In addition, the reactive groups are concentrated in a polymer coil even in a dilute solution. The kinetics of such reactions in the polymer system have attracted an interest of photochemists. Shindo et al. have investigated the photodimerization between cinnamoyl groups attached to polymethylmethacrylate (PMMA) side chains. ${ }^{1-3)}$ Horie et al. have studied the kinetics of photodimerization between anthryl groups attached to polystyrene side chains with eosinyl groups as fluorescent probes. ${ }^{4,5)}$ In this study, we have synthesized PMMA with anthryl groups in the part of the side chains, and discuss the kinetics of the photodimerization of the anthryl groups in dilute solutions. The volumes of polymers before and after reaction were estimated by GPC and dynamic light scattering measurements.

\section{Experimental}

Samples

PMMAs with anthryl groups in the part of the side chains were synthesized by copolymerizing 9-anthrylmethyl methacrylate (AMMA) with methyl methacrylate(MMA) at various ratios using AIBN initiator. Scheme I represents the structures of copolymer and a monomer-like model 
compound, 9-anthrylmethyl isobutylate (AMIB). The molar fractions (x) of AMMA in the copolymers used in the present study were $0.05,0.15$, and 0.2 .

\section{Measurements}

A $3 \mathrm{ml}$ portion of the polymer solution in THF were placed in a rectangular quartz cell, degassed under vacuum and irradiated through a $320-420 \mathrm{~nm}$ bandpass filter with a $150 \mathrm{~W}$ Xe lamp as a light source. The extent of the photodimerization was determined with the absorbance at $367.5 \mathrm{~nm}$.

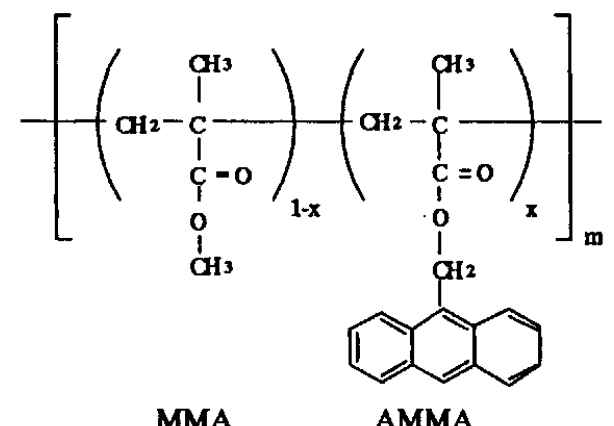

A: Copolymer<smiles>CC(C)C(=O)OCOCc1c2ccccc2cc2ccccc12</smiles>

AMIB

B: Model compound

Scheme I Chemical structures of copolymer and

low molecular weight model compound.

\section{Results and Discussion}

\section{Photodimerization}

Figure 1 shows the changes in absorption spectra of the copolymer $(x=0.15)$ in THF with UV-irradiation time. The absorbance of the band in the range of 300-400 nm, which is attributed to $\pi-\pi *$ transition, is decreased with UV-irradiation time. Figure 2 illustrates the changes in absorbances at $367.5 \mathrm{~nm}$ for three copolymers and AMIB (as a model compound) with UV-irradiation time. In contrast to AMIB, the absorbances of the copolymers were appreciably decreased in the initial stage of the reactions.

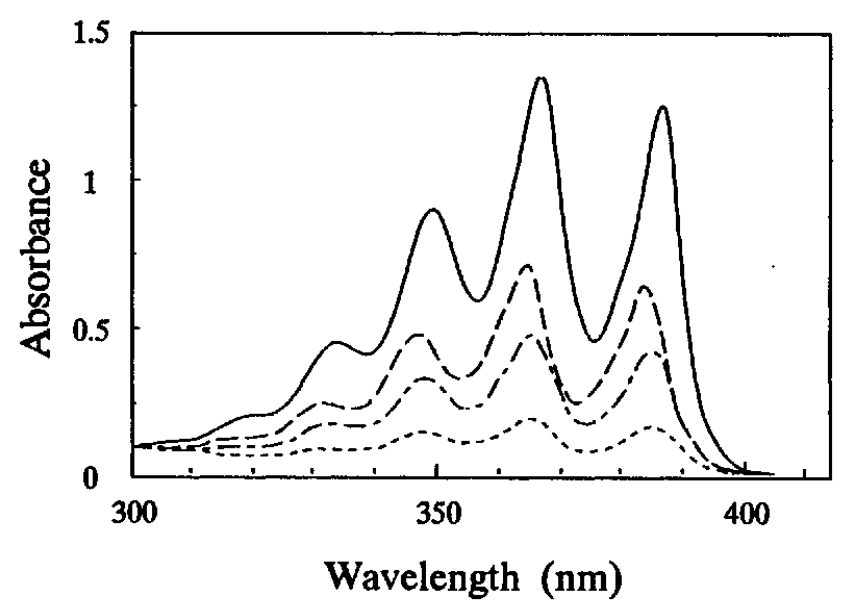

Fig.1 Absorption spectra of copolymer of AMMA and MMA in THF solution at various irradiation times (min): 


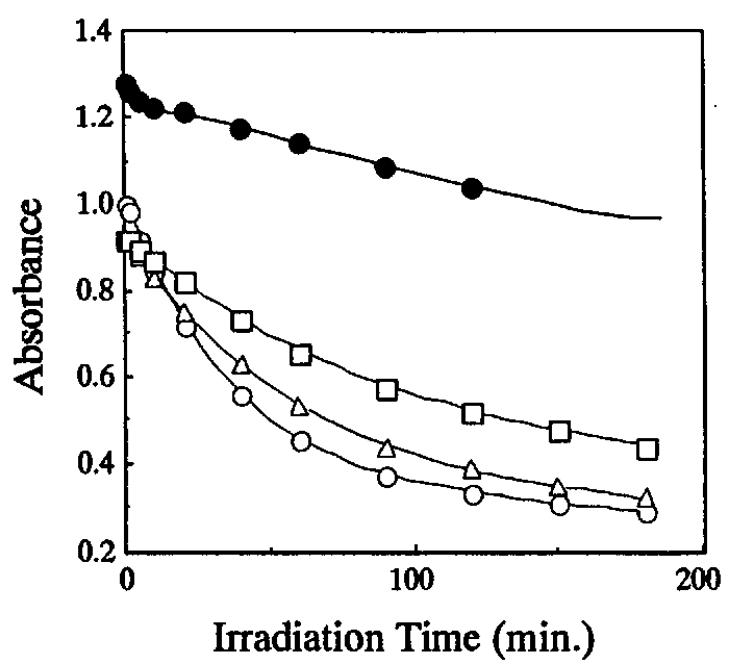

Fig.2 Changes in absorbance at $367.5 \mathrm{~nm}$ for AMIB $(0)$ and copolymers of various molar fractions of AMMA. $x=0.2(0) ; 0.15(\Delta) ; 0.05(\square)$.

The efficiency Cpd of conversion of anthracene molecules to photodimer was estimated by Cpd $(\%)=(1-A(t) / A(0)) \times 100$, where $A(t)$ refers to the absorbance of anthryl groups at $367.5 \mathrm{~nm}$ at a UV-irradiation time t. The changes in Cpd with UV-irradiation time are shown in Fig.3.

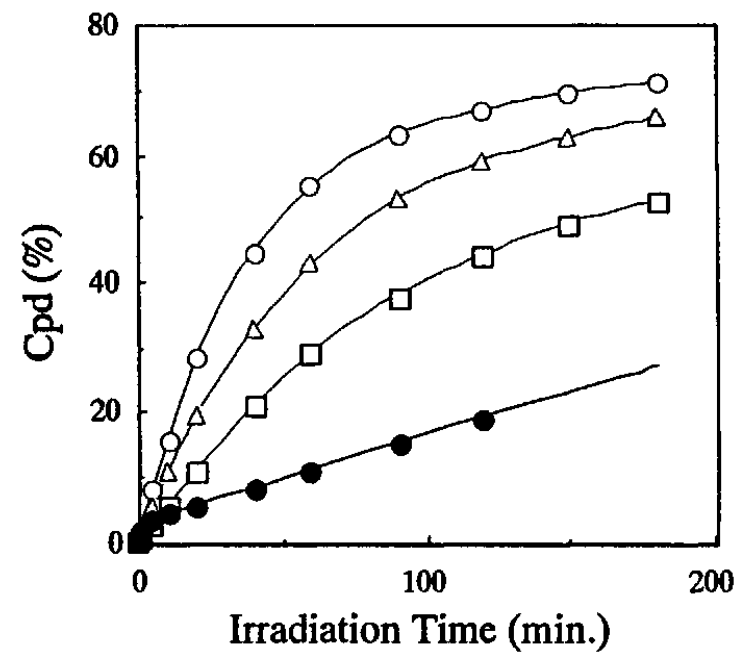

Fig.3 Changes in conversion for AMIB (O) and copolymers of various molar fractions of AMMA. $\mathrm{x}=0.2(\mathrm{O}) ; 0.15(\triangle) ; 0.05(\square)$.

Cpd $(\%)=(1-A(t) / A(0)) \times 100$

The results indicated that the higher AMMA contents in the copolymers, the faster the photodimerization of anthryl groups in polymer chains was proceeded, and the earlier the equilibrium in the reaction was attained. The rise in Cpd for AMIB is very slow, compared to the 
copolymers, and then after more than $50 \mathrm{hr}$ the equilibrium was attained. The saturated values of Cpd for copolymers with $x=0.05,0.15$, and 0.2 , were 81,77 , and $76 \%$, respectively, whereas the one for AMIB was $97 \%$.

The incomplete conversion for the copolymers is inferred to come from the followings : As the intramolecular crosslinkages due to photodimerization of anthracenes progresses, some anthracenes are unable to be dimerized because of the restriction of the motions of polymer main chain.

Photodimerization of anthracenes, which is induced by the collision between anthracenes in exited state and in ground state, is subjected to a second order kinetics, on the assumption that the concentration of anthracenes in the exited state is proportional to that in the ground state. Figure 4 shows that the photodimerization of AMIB obeys sufficiently the second-order kinetics.

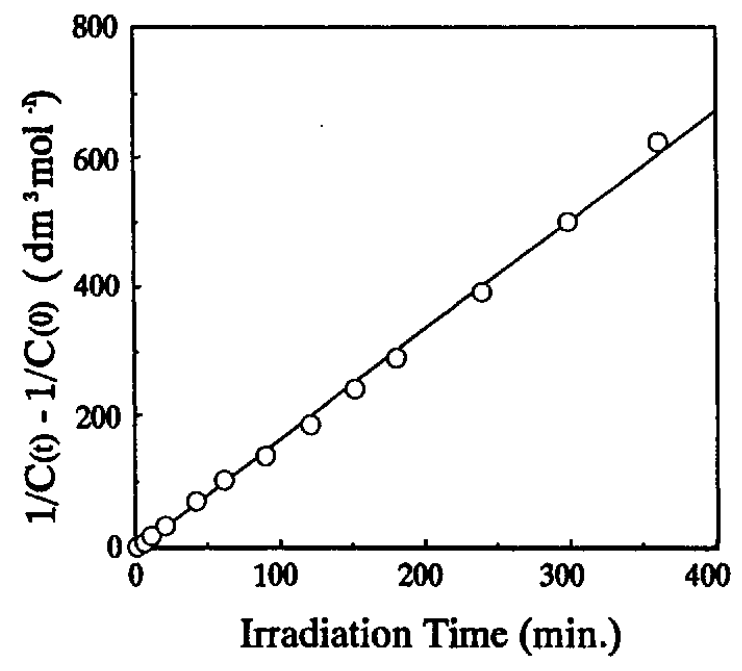

Fig.4 Second-order plots for photodimerization of Anthracene in AMIB.

Moreover, the second-order plots for the copolymers with $x=0.05,0.15$, and 0.2 are shown in Fig.5.

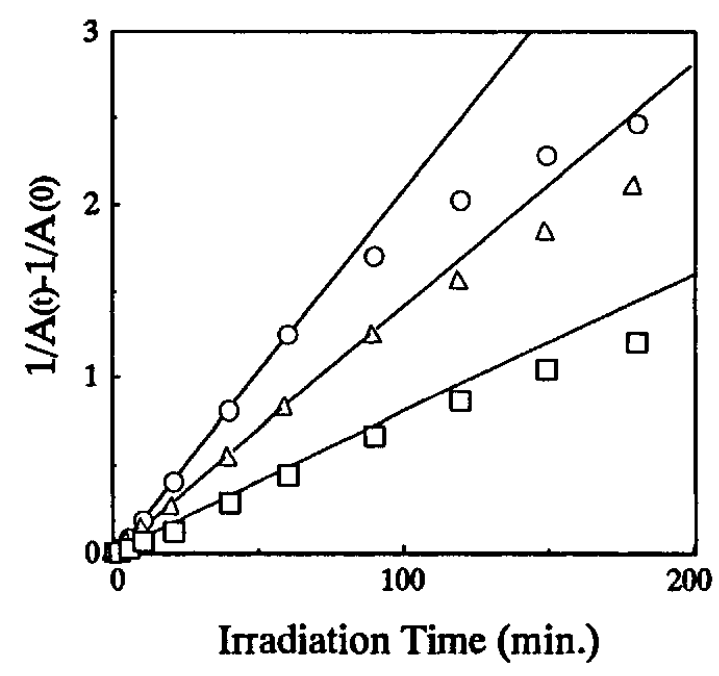

Fig.5 Plots of $1 / A(t)-1 / A(0)$ of copolymers against 
At the initial stage of the reaction, the plots are on straight lines, indicating that the photodimerization follows a second-order kinetics. However, as the reactions proceed, the plots are deviated from straight lines, indicating that the reaction rate is changed to the lower rates.

The critical overlap concentration of the copolymers, where polymer coils collide each other, is estimated to be $14.8 \mathrm{~g} /$, using the value of Stokes radius for the copolymer with $\mathrm{x}=0.15$ was $15.8 \mathrm{~nm}$, which was measured by means of dynamic light scattering. The experimental concentration of the samples are much smaller than the critical overlap concentration. Therefore, it is suggested that the photodimerization occurs within an individual polymer coil. The concentrations of anthracenes within an individual polymer coil were calculated from Stokes radius and the number of anthracenes in one polymer coil. Then, the second-order plots for the copolymers and AMIB were redepicted as shown in Fig.6, based on the concentrations of anthracenes within an individual polymer coil. For the copolymers, at the initial stage of the reaction, the plots are on the same line, regardless of the AMMA contents in the copolymers. The rate constants of AMMA and the copolymers at the initial stage are determined to be $\mathrm{K}_{\mathrm{A}}=0.0264$ and $\mathrm{K}_{\infty}=0.0148 \mathrm{dm}^{3} \mathrm{~mol}^{-1} \mathrm{~s}^{-1}$, respectively. The results indicate that even at the initial stage, the motions of anthracenes attached to side chains of the copolymers are significantly restricted by the main chain, compared to unperturbed motion of AMIB.

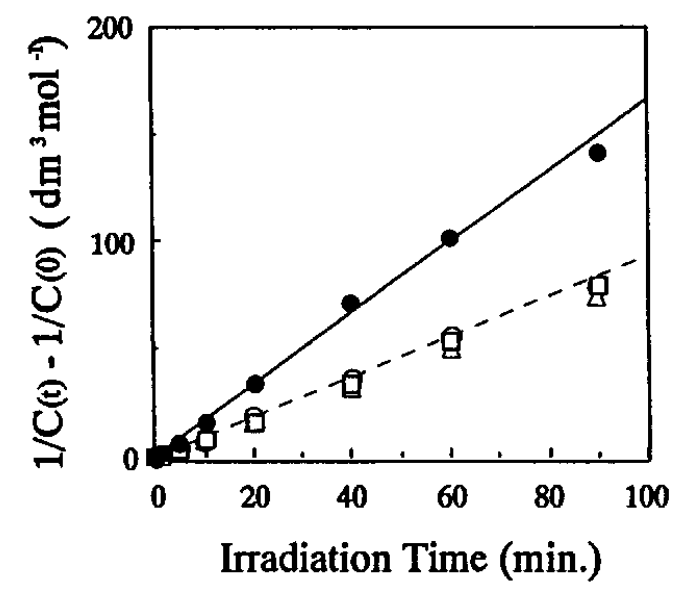

Fig.6 Plots of $1 / C(t)-1 / C(0)$ of AMIB ( $d$ and copolymers against irradiation time. $x=0.2(O) ; 0.15(\Delta) ; 0.05(\square)$.

\section{GPC Measurement}

GPC patterns as a function of UV-irradiation time for the copolymer with $\mathrm{x}=0.15$ are shown in Fig. 7. An appreciable change in the chromatogram was not recognized until an irradiation time of $120 \mathrm{~min}$, and then the elution pattern indicated a drastic change that the peak retention time was shifted to the longer time. This result implies that at the initial stage of the reaction, the photodimerization occurs between anthryl groups in the short ranges, and then occurs in the long ranges and thus an appreciable structural-shrinkage of the copolymer is induced by the intra-crosslinkages due to photodimerization of anthracenes. 


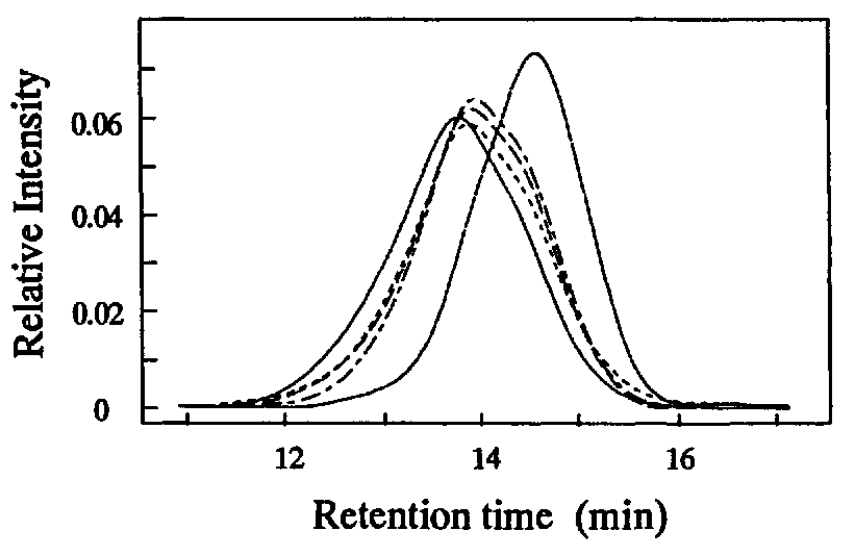

Fig.7 GPC chromatograms of copolymer $(x=15)$ at various irradiation times $(\mathrm{min}):-, 0$; $\cdots, 40$; - - , 60; - - , 120; -180 .

\section{Conclusion}

The photodimerization of anthracenes attached to the side chain in the polymer coil obeys second order kinetics at the initial stage of the reaction, but as the polymer coil shrinks the mobility of contour is restricted and reaction is suppressed.

\section{References}

1) Y. Shindo, K. Horie, and I. Mita, Chem. Lett., (1983) 639.

2) Y. Shindo, H. Sato, T. Sugimura, K. Horie, and I. Mita, Eur. Polym. J., 25 (1989) 1033.

3) Y. Shindo, T. Sugimura, Netsukoukaseijushi, 11 (1990) 219.

4) T. Torii, H. Ushiki and K. Horie, Polym. J., 24 (1992) 1057.

5) T. Torii, H. Ushiki and K. Horie, Polym. J., 25 (1993) 173. 